\title{
El nexo agua-energía en plantas termoeléctricas
}

Fecha de publicación: 18 de julio de 2021

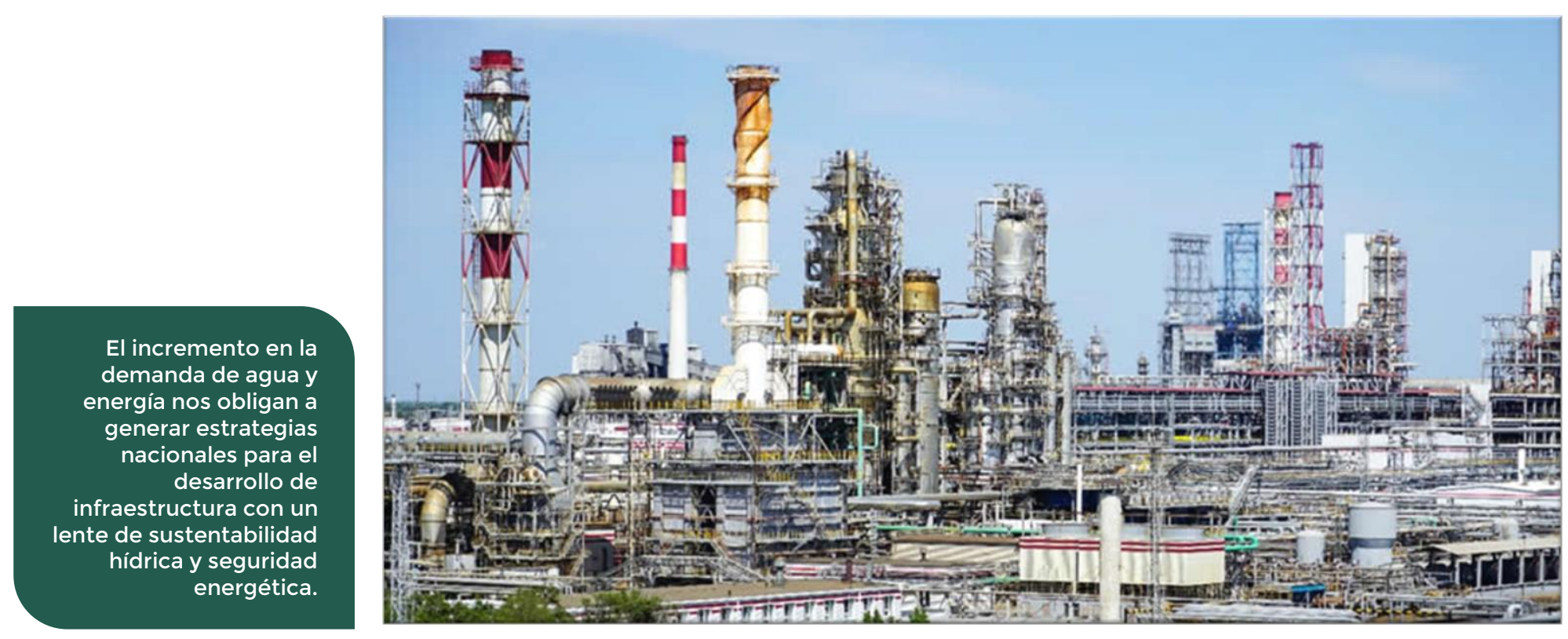

La relación entre agua y producción de energía es tan íntima, que se presenta de diversas formas como resultado de la necesidad de grandes cantidades de energía para bombear, tratar, transportar, calentar, enfriar y reciclar agua, al mismo tiempo que el agua es necesaria para generar el vapor que produce electricidad a través del movimiento de turbinas, y es el fluido principal para el enfriamiento de las plantas termoeléctricas de todo el mundo. Esta interdependencia entre agua y energía implica nuevos retos para los tomadores de decisiones, cuyo objetivo es garantizar un futuro con abasto seguro y sostenible de ambos insumos (Dai et al., 2018).

La generación de energía eléctrica en plantas termoeléctricas es una de las actividades en las que la revisión del nexo agua-energía es de suma importancia, dada la alta dependencia que tiene su funcionamiento sobre la disponibilidad de agua que se utiliza en los sistemas de enfriamiento. Estos sistemas constituyen, dentro de las plantas termoeléctricas, la parte más intensiva desde el punto de vista hídrico (Sovacool y Gilbert, 2014) y, por tanto, la que presenta mayores oportunidades de mejora para reducir la huella hídrica azul de esta industria. Este hecho es particularmente importante en países con dos características: por un lado, en los que la generación de energía a través de este tipo de sistemas desempeña un papel determinante en la producción total de energía, y en los que la escasez regional de agua es preocupante. Tal es el caso de los Estados Unidos de América (Unesco,2014) y China (Zhang y Anadon, 2013, Zhang et al., 2014).

De acuerdo con datos de la Agencia Internacional de Energía es posible atribuir a la producción primaria de energía eléctrica aproximadamente el $10 \%$ del volumen total de extracciones de agua en todo el 
mundo (IEA, 2016). En Estados Unidos de América y en Europa Occidental, cerca del 50 \% del volumen de extracciones de agua es utilizado para la producción de energía eléctrica, siendo el uso principal el sistema de enfriamiento (EEA, 2009), mientras que en China este número asciende aproximadamente al $84 \%$ del volumen total de extracciones de agua (Qin et al., 2015).

Reportes globales indican un incremento en la escasez de agua en diversas regiones del mundo, por lo que cada vez es más necesario evaluar las necesidades hídricas a futuro del sector energético y, en particular, de la generación a través de plantas termoeléctricas en varios países. Así lo han hecho el Reino Unido (Murrant et al, 2017) y Sudáfrica (Thopil y Pouris, 2016). Después de ser utilizado en los sistemas de enfriamiento de las plantas termoeléctricas, el efluente de agua utilizada requiere diversos procesos de tratamiento que consumen energía adicional antes de ser descargadas para su utilización posterior. En los últimos años, esto ha redundado en un incremento en el estudio de la relación aguaenergía dentro de las plantas termoeléctricas (Sanders, 2015), lo que ha dado lugar a muchos esfuerzos enfocados a evaluar y optimizar las eficiencias de uso de agua y energía a través de distintas estrategias, como la configuración en serie y paralelo de los enfriadores (temperatura de retorno y caudal) (Sun et al., 2015), sistemas basados en algoritmos no lineales (Salazar et al., 2011) y los índices de riesgo a la sustentabilidad (Roy et al., 2012).

Las fuentes de abastecimiento de agua para las plantas termoeléctricas incluyen las superficiales, las subterráneas y aquellas provenientes de un tratamiento para su reutilización. El uso no consuntivo del agua que se da en estas plantas se define como aquel volumen removido de una fuente de abastecimiento natural y que es devuelto a la misma después de su uso. Mientras que el uso consuntivo implica un consumo, porque el agua que es extraída de la fuente no es devuelta a la misma, y se pierde (ya sea a través de la evaporación o de la incorporación del agua en un producto). Aproximadamente, cerca del 80 \% de la energía eléctrica global se genera mediante plantas termoeléctricas (usando combustibles fósiles y energía nuclear) (IEA, 2017). Así, el agua es un elemento crucial en la generación de energía termoeléctrica, y su desempeño recae directamente sobre los sistemas de enfriamiento, lo que los hace usuarios hídricos muy demandantes, pues tienen la necesidad de acceso a grandes volúmenes de agua para su buen funcionamiento.

Por otro lado, la energía representa un elemento imperativo en la extracción, tratamiento, potabilización y distribución del agua. La energía utilizada para los sistemas de abastecimiento ha crecido rápidamente, debido al pronunciado incremento en la demanda por este servicio en todo el mundo. De hecho, se anticipa que la demanda de agua crezca hasta en un 55 \% para 2050, en comparación con los niveles registrados en el año 2000 (OECD, 2012). Estudios globales indican que cerca del $10 \%$ de las ventas de energía son específicamente para la operación de los sistemas hídricos (sean de abastecimiento o saneamiento) (Liu 2016). Para el año 2040 se proyecta un requerimiento de energía de más del doble para el sector hídrico global. A manera de ejemplo, dentro de una planta de tratamiento de aguas residuales municipales existen estimaciones de que el 57 \% de la energía se usa para la aireación, 30 \% para el procesamiento de biosólidos y el 15 \% para el bombeo (Pabi, 2013).

Históricamente, la utilización del agua como fluido principal en los sistemas de enfriamiento de las plantas termoeléctricas se debe a su alta disponibilidad y fácil acceso, sin embargo, su transporte hacia las torres o enfriadores requiere una gran cantidad de energía. Los sistemas de enfriamiento están compuestos por dos subsistemas (una red de enfriadores y una red de bombeo) que son optimizados de forma separada. En este sentido, existen diversas estrategias de mejoramiento de la eficiencia energética e hídrica dentro de las operaciones de enfriamiento. Estas incluyen el incremento en el reciclaje del agua y la recaptura y reutilización de la energía dentro de la planta. La mayor parte de la 
energía se pierde por fricción y caídas de presión, o es desechada en pérdidas de calor o salmueras concentradas. Por ejemplo, actualmente se cuenta con diversos materiales que permiten mejorar la transferencia de calor y reducir la fricción, con lo que se incrementa la eficiencia energética hasta en un 10 \% (Zhang et al., 2017). Para las operaciones en torres de enfriamiento, la forma más sencilla de reducir el uso de agua es a través de la instalación de sistemas de recirculación que reutilicen o reciclen el agua. Sin embargo, esto requiere una alta inversión económica en la modificación de los sistemas, ya que tan solo en EUA el 40 \% de las plantas tienen sistemas de un solo circuito, sin recirculación (Gude, 2015). Otras estrategias de reutilización incluyen el uso de aguas municipales tratadas para el enfriamiento, opción que no necesariamente reduce la huella hídrica de la planta, pero sí reduce las extracciones de agua de las fuentes de abastecimiento naturales, además de ser más económica respecto a la instalación de plantas desaladoras. El reto para ello consiste en instalar trenes de tratamiento mejorados para atender el carbón orgánico total y la demanda química de oxígeno, así como la subsecuente remoción de minerales, ya que existen especificaciones de calidad del agua para los influentes de estos sistemas (Hill et al., 2014).

El incremento en la demanda de agua y energía nos obligan a generar estrategias nacionales para el desarrollo de infraestructura con un lente de sustentabilidad hídrica y seguridad energética. La oportunidad para las plantas termoeléctricas estriba claramente en el mejoramiento de sus eficiencias hídrica y energética, ya sea a través de la instalación de sistemas de tratamiento específicos para la planta, que nos permitan garantizar la calidad de agua necesaria para los sistemas de enfriamiento, o del reacondicionamiento de los sistemas de enfriamiento para promover la recirculación del agua o la instalación de circuitos líquidos (Scanlon et al., 2013). Para todo ello se requiere una acción conjunta de diversos sectores, así como del uso de la evidencia científica y la tecnología que nos permita avanzar como nación en el aseguramiento del agua y la energía para las generaciones presentes y futuras.

Referencias

Dai, J., Wu, S., Han, G., Weinberg, J., Xie, X., Wu, X., Song, X., Jia, B., Xue, W., Yang, Q., 2018. Water-energy nexus: A review of methods and tools for macroassessment. Appl. Energy 210, 393-408.

EEA, 2009. Water Resources Across Europe: Confronting Water Scarcity and Drought. European Environment Agency, Copenhagen.

Gude, V.G., 2015. Energy and water autarky of wastewater treatment and power generation systems. Renew. Sustain. Energy Rev. 45, 52-68.

Hill, A., Hayes, T., Sishtla, C., 2014. Reclamation of Wastewater for Cooling Tower Operations. Gas Technology Institute.

IEA, 2016. Water Energy Nexus, Excerpt from the World Energy Outlook 2016. OECD/IEA (International Energy Agency), France, p. 13.

IEA, 2017. Key World Energy Statistics. OECD, International Energy Agency, France, p. 100.

Liu, Y., Hejazi, M., Kyle, P., Kim, S.H., Davies, E., Miralles, D.G., Teuling, A.J., He, Y., Niyogi, D., 2016. Global and regional evaluation of energy for water. Environ. Sci. Technol. 50, 9736-9745.

Murrant, D., Quinn, A., Chapman, L., Heaton, C., 2017a. Water use of the UK thermal electricity generation fleet by 2050: Part 1 identifying the problem. Energy Policy 108, 844-858.

OECD, 2012. OECD Environmental Outlook to 2050: The Consequences of Inaction. Organization for Economic Co-operation and Development (OECD), Paris.

Pabi, S., 2013. Electricity Use and Management in the Municipal Water Supply and Wastewater Industries. EPRI, Palo Alto, CA. 
Qin, Y., Curmi, E., Kopec, G.M., Allwood, J.M., Richards, K.S., 2015. China's energy water nexus assessment of the energy sector's compliance with the " 3 RedLines" industrial water policy. Energy Policy 82, 131-143.

Roy, S.B., Chen, L., Girvetz, E.H., Maurer, E.P., Mills, W.B., Grieb, T.M., 2012. Projecting water withdrawal and supply for future decades in the U.S. under climate change scenarios. Environ. Sci. Technol. 46, 25452556.

Salazar, J.M., Zitney, S.E., Diwekar, U.M., 2011. Minimization of water consumption under uncertainty for a pulverized coal power plant. Environ. Sci. Technol. 45, 4645-4651.

Sanders, K.T., 2015. Critical review: uncharted waters? The future of the electricity water nexus. Environ. Sci. Technol. 49, 51-66.

Scanlon, B.R., Reedy, R.C., Duncan, I., Mullican, W.F., Young, M., 2013. Controls on water use for thermoelectric generation: case study Texas, US. Environ. Sci. Technol. 47, 11326-11334.

Sovacool, B.K., Gilbert, A., 2014. Developing adaptive and integrated strategies for managing the electricity-water nexus. Univ. Richmond Law Rev. 48, 997-1032.

Sun, J., Feng, X., Wang, Y., 2015. Cooling-water system optimisation with a novel two-step sequential method. Appl. Therm. Eng. 89, 1006-1013.

Thopil, G.A., Pouris, A., 2016. A 20-year forecast of water usage in electricity generation for South Africa amidst water scarce conditions. Renew. Sustain. Energy Rev. 62, 1106-1121.

UNESCO, 2014. The United Nations World Water Development Report 2014: Water and Energy, France. Zhang, C., Anadon, L.D., 2013. Life cycle water use of energy production and its environmental impacts in China. Environ. Sci. Technol. 47, 14459-14467.

Zhang, C., Anadon, L.D., Mo, H., Zhao, Z., Liu, Z., 2014. Water-carbon trade-off in China's coal power industry. Environ. Sci. Technol. 48, 11082-11089.

Zhang, S., Zeng, X., Igartua, A., Rodriguez-Vidal, E., van der Heide, E., 2017. Texture design for reducing tactile friction independent of sliding orientation on stainless steel sheet. Tribol. Lett., 65 